\title{
MATCHING DE IDENTIDADE COM ESTÍMULOS COMPOSTOS E A EMERGÊNCIA DE RELAÇÕES SIMBÓLICAS ENVOLVENDO MÚSICA CLÁSSICA E EXPRESSÕES FACIAIS
}

\section{IDENTITY MATCHING WITH COMPOUND STIMULI AND THE EMERGENCE OF SYMBOLIC RELATIONS IN CLASSICAL MUSIC AND FACIAL EXPRESSIONS}

\author{
ELOÍSA DE SOUZA FERNANDES - ORCID 0000-0001-6884-4902 \\ JULIANA YU RIBEIRO TOYODA - ORCID 0000-0002-5354-2167 \\ NATASHA TAKUNO HESPANHOL - ORCID 0000-0002-1045-3017 \\ VIVIANE NOGUEIRA DA SILVA - ORCID 0000-0002-9335-622X \\ ALCEU MARTINS FILHO - ORCID 0000-0002-4090-224X \\ PAULA DEBERT - ORCID 0000-0002-2519-2569 \\ INSTITUTO dE PSICOLOGIA, UNIVERSIDADE DE SÃo PAULO \\ InStituto NACIONAL dE CIÊnCIA E TECNOlogia SOBRE COMPORTAMENTO COGNIÇÃo E ENSINO
}

\begin{abstract}
RESUMO
O objetivo do presente experimento foi verificar a possibilidade de se estabelecer relações condicionais emergentes entre o nome de um compositor de música clássica, seu respectivo retrato e um trecho de uma de suas músicas após treino com o procedimento de matching de identidade (IMTS) com estímulos compostos. Um objetivo adicional consistiu em averiguar a quais expressões faciais os participantes relacionariam os nomes dos compositores e os títulos das obras. Quatro voluntários foram submetidos ao IMTS com estímulos compostos (AB-AB). Os estímulos eram nome do compositor e título da obra (A), retrato do compositor (B), trecho de oito segundos da obra (C), e foto de expressão facial de emoção (D). Foram testadas as relações arbitrárias A-B, B-A, AC-B, A-C e A-D. Para três dos quatro participantes, as relações condicionais arbitrárias emergentes foram estabelecidas. Além disso, observou-se que apenas esses três participantes estabeleceram relações sistemáticas entre as expressões faciais e os nomes dos compositores/títulos da obra. O procedimento investigado produziu relações emergentes que envolveram trechos de músicas como estímulos e, também, permitiu estabelecer relações entre nome de compositores e expressões faciais sem treino direto. Sendo assim, é um procedimento eficaz para ensino e produção de relações emergentes entre estímulos sonoros mais complexos e pode permitir transferência do "significado" de músicas para novos estímulos.
\end{abstract}

Palavras-chave: matching de identidade, estímulos compostos, música clássica, expressões faciais.

\begin{abstract}
The present experiment aimed to verify the possibility of establishing emergent conditional relations between the name of a composer of classical music, his respective portrait and an excerpt from one of his songs after training with the Identity matching-to-sample procedure (IMTS) with compound stimuli. An additional objective of this experiment was to verify which facial expressions the participants would relate to the names of the composers and title of the work. Four volunteers were submitted to identity matching with compound stimuli (AB-AB). The stimuli were the name of the composer and title of the work (A), portrait of the composer (B), eight-second excerpt of the music (C), and facial expression picture that would represent emotion (D). The arbitrary relations A-B, B-A, AC-B, A-C and A-D were tested. For three of the four participants, the emergent arbitrary conditional relations were established. In addition, it was observed that only these three participants established systematic relations between the facial expressions and the names of the composers/titles of the work. The procedure under investigation produced emergent relations that involved excerpts of music as stimuli and, also, allowed to establish relations between composers' names and facial expressions without direct training. Thus, it is an effective procedure for teaching and producing emerging relationships between more complex auditory stimuli and may allow transferring the "meaning" of songs to new stimuli.
\end{abstract}

Key words: identity matching, compound stimuli, classical music, facial expressions.

Paula Debert é pesquisadora do Instituto Nacional de Ciência e Tecnologia sobre Comportamento, Cognição e Ensino com financiamento do CNPq (Processo 465686/2014-1) e da FAPESP (Processo 2014/50909-8), Alceu Martins Filho é financiado pela bolsa CNPq (Processo 166325/2018-0). Correspondências referente a este artigo devem ser enviadas a pdebert@uol.com.br

DOI. 10.18542/rebac.v14i1.7156 
Um procedimento utilizado para estabelecer classes de estímulos equivalentes é o Matching-To-Sample (MTS). O MTS padrão envolve a apresentação de um estímulo-modelo (estímulo condicional) e dois ou mais estímulos-escolha (estímulos discriminativos) a cada tentativa. Apenas a resposta de selecionar o estímuloescolha designado pelo experimentador como relacionado ao estímulo-modelo apresentado a cada tentativa é seguida de reforço. O treino dessas relações condicionais envolvendo, por exemplo, os estímulos do conjunto A como modelo e do conjunto B como escolha, bem como do conjunto $\mathrm{B}$ como modelo e do conjunto $\mathrm{C}$ como escolha, podem promover a emergência de novas relações sem treino direto como, por exemplo, a relação entre A e C (e.g., Sidman, 1994, 2000).

Os estudos, em geral, utilizam o MTS com estímulo-modelo e estímulo-escolha unitários. Porém, algumas pesquisas têm indicado a produção de relações de equivalência a partir de treinos com estímulo-modelos e/ou estímulo-escolhas compostos (e.g., Markham \& Dougher, 1993; Rebello et al., 2010; Salvatori, Silva, Belem, Modenesi, \& Debert, 2012; Schenk, 1993, 1995; Stromer \& Stromer, 1990). Uma variação do procedimento MTS é o matching de identidade (IMTS - Identity Matching-To-Sample). Nesse procedimento, as respostas de escolha do estímulo-escolha igual ao estímulo-modelo são seguidas de reforço (e.g., Cumming \& Berryman, 1965).

Schenk (1993) utilizou o IMTS com estímulos compostos. Nesse procedimento, tanto o estímulo-modelo quanto os estímulos-escolha eram compostos por dois elementos $(\mathrm{AB})$. A cada tentativa era apresentado um estímulo-modelo composto e três estímulos-escolha compostos. Apenas um dos três estímulos-escolha era igual ao estímulo-modelo apresentado em cada tentativa. Após estabelecidas essas relações de identidade entre estímulos compostos (AB-AB), foram conduzidos testes, em extinção, e os estímulos foram apresentados em sua forma unitária, avaliando a emergência de relações condicionais arbitrárias A1-B1, A2-B2, A3-B3, B1-A1, B2-A2 e B3-A3. Em um teste posterior, um novo estímulo-modelo composto foi apresentado a cada tentativa (AC) e os três estímulos-escolha foram B1, B2 e B3. Por fim, foram conduzidos testes adicionais com estímulos unitários (C-B, B-C, A-C e C-A). Os resultados mostraram que seis dos oito participantes apresentaram desempenhos emergentes consistentes com o que foi treinado de forma que poder-se-ia afirmar que foram estabelecidas três classes de equivalência (A1B1C1, A2B2C2 e A3B3C3).

Em uma replicação do estudo de Schenk (1993; 1995), o estudo de Rebello et al. (2010) apontou que um treino com IMTS com estímulos compostos foi capaz de produzir relações condicionais arbitrárias emergentes envolvendo ideogramas japoneses (estímulo B) e suas respectivas palavras escritas em português (estímulo A) e ideogramas japoneses falados (estímulo C). Os seis participantes do estudo foram submetidos ao treino com o IMTS com estímulos compostos (AB-AB) e aos testes AB, B-A, AC-B, C-A, C-B. Os resultados mostraram que os seis participantes apresentaram a emergência das relações testadas. Rebello et al. (2010), então, concluíram que o IMTS é um procedimento eficaz de aprendizagem sem erros para estabelecer relações emergentes entre palavras em português e seus respectivos ideogramas japoneses.

O experimento de Salvatori et al. (2012), também, envolveu uma replicação do estudo de Schenk (1993) e apresentou resultados importantes com implicações para o ensino de habilidades musicais. $\mathrm{O}$ objetivo desse experimento foi verificar a possibilidade de produzir relações condicionais emergentes envolvendo notas musicais escritas (estímulo A), representações gráficas das notas musicais na pauta (estímulo B) e estímulos auditivos tocados no piano (estímulo C). Foi realizado, ainda, um teste para comprovar a generalização para os estímulos auditivos tocados na flauta (estímulo C'). A pesquisa contou com cinco participantes sem nenhum conhecimento prévio sobre relações entre sons de notas musicais e suas representações gráficas. Os estudantes foram submetidos ao treino de IMTS com estímulos compostos $\mathrm{AB}-\mathrm{AB}$ e, posteriormente, aos testes A-B, AC-B, C-A e C'-A. Para quatro dos cinco estudantes houve a emergência de todas as relações testadas, permitindo afirmar que o treino de IMTS com estímulos compostos é efetivo para estabelecer relações condicionais emergentes envolvendo notas musicais.

O presente experimento pretendeu estender os achados de Salvatori et al. (2012) no sentido de avaliar o potencial do procedimento IMTS para estabelecimento de relações condicionais emergentes envolvendo estímulos sonoros mais complexos, como trechos de música. Para tanto, foram realizados os treinos com IMTS e os testes descritos anteriormente. Os estímulos utilizados foram: nome de um compositor de música clássica/título da obra (A), seu respectivo retrato (B) e um trecho da música (C). Os estímulos sonoros empregados no presente estudo (trechos das música) envolveram várias notas e tiveram duração mais prolongada do que as notas musicais breves apresentadas no experimento de Salvatori et al. (2012). Portanto, a eventual identificação da emergência de relações condicionais envolvendo esses estímulos mostraria um impacto maior do procedimento analisado na medida em que permitirá o desenvolvimento de tecnologias de ensino de repertórios complexos que envolvem, por exemplo, harmonia, melodia, ritmo, entre outros.

Um objetivo adicional do presente estudo foi verificar se os participantes relacionariam cada um dos nomes de compositores de música clássica/título da obra (estímulo A) a uma expressão facial específica (D) a partir de um teste A-D. O estudo de Bortoloti e de Rose (2007) mostrou que é possível que estímulos abstratos sejam avaliados mais ou menos positivamente sem terem sido diretamente relacionados a expressões faciais específicas, apenas por fazerem parte da mesma classe de equivalência que essas expressões (alegre, raivosa ou neutra). Os autores argumentaram que os "significados" das expressões faciais se transferiram para os estímulos abstratos que fazem parte da mesma classe de equivalência que a expressão facial mesmo sem treino direto. Tendo em 
vista a possibilidade do "significado" das expressões faciais se transferirem para os estímulos abstratos (Bortoloti \& de Rose, 2007), os testes adicionais ora propostos pretendem fornecer informações iniciais para avaliar se eventuais "significados" dos trechos das músicas apresentados se transfeririam aos estímulos As (nomes de compositor de música clássica/título da obra), permitindo que expressões faciais específicas diferentes fossem escolhidas na presença de cada estímulo A. O estabelecimento de relações sistemáticas entre estímulos As e as expressões faciais específicas (estímulos Ds) corroboraria a demonstração do estabelecimento de classes de equivalência via transferência de função.

\section{MÉTODO}

\section{Participantes}

Participaram do experimento quatro alunos do primeiro semestre do curso de graduação em Psicologia, com idade entre 17 e 23 anos, sem contato prévio com Análise Experimental do Comportamento e/ou pesquisas envolvendo o procedimento de IMTS e com pouco ou nenhum contato com música clássica. Essa última informação foi adquirida pelo relato verbal do participante no momento do recrutamento, por meio da pergunta "Você possui conhecimentos sobre música clássica?". Foi requisitado que os participantes assinassem um Termo de Consentimento Livre e Esclarecido (TCLE) e uma carta de informação contendo a descrição geral do ambiente e das condições de aplicação do experimento, sem que houvesse a descrição do procedimento a ser realizado. A pesquisa foi aprovada pelo Comitê de Ética em Pesquisa com Seres Humanos submetido por meio da Plataforma Brasil (CAAE: 57271916.5.0000.5561).

\section{Equipamento}

As sessões foram realizadas em uma sala silenciosa com dimensões de $4 \mathrm{~m}$ x $3 \mathrm{~m}$. Utilizou-se um computador IBM® com monitor de $256 \mathrm{~mm}$ (14 polegadas) equipado com o programa em linguagem Visual Basic $®$ para apresentação dos estímulos, o registro e a organização dos dados.

\section{Estímulos}

Os estímulos utilizados foram três nomes de compositores juntamente com o nome de uma de suas músicas (estímulos As), três imagens dos compositores (estímulo Bs), três áudios de trechos de 8s de uma de suas músicas (estímulos Cs) e três fotos de diferentes expressões faciais universais (estímulos Ds) - ver Tabela 1. A duração dos trechos de áudios foi definida conforme sugerido por Bachorik et al. (2009). As três expressões faciais utilizadas (D1 - expressão de felicidade, D2 expressão de tristeza e D3 - expressão neutra) são procedentes de um subconjunto da Radboud Faces Database - RaFD (Langner et al., 2010), correspondente a faces de modelos caucasianos adultos, com olhar direto, tiradas de um ângulo de câmera de $90^{\circ}$. Os modelos vestem uma camiseta preta e não usam nenhum tipo de acessório ou o cabelo sobre o rosto (Tabela 1). Essa base de dados foi escolhida por apresentar aspectos técnicos padronizados e por contar com um nível alto de reconhecimento das expressões faciais pretendidas, segundo a validação do estudo. As expressões faciais de felicidade e tristeza foram utilizadas por serem diferenciadas e classificadas com maior precisão em estudos que empregaram trechos de música (e.g., Mohn, Argstatter, \& Wilker, 2011).

\section{Procedimento}

O procedimento envolveu seis fases. Inicialmente, foram selecionados apenas os participantes que não tinham contato com música clássica como descrito anteriormente. Na Fase 1 (Treino AB-AB), foram treinadas as relações de identidade entre os estímulosmodelo compostos $\mathrm{AB}$ e os estímulos-escolha compostos $\mathrm{AB}$ formados por nome do compositor e imagem do compositor. Nas demais fases, foram realizados testes de relações emergentes para que se pudesse verificar os desempenhos emergentes produzidos pelo treino conduzido na Fase 1. Na Fase 2 (Teste A-B), foram verificadas as relações emergentes entre os estímulosmodelo unitários A e os estímulos-escolha unitários B. Na Fase 3 (Teste B-A), foram verificadas as relações emergentes entre os estímulos-modelo unitários $\mathrm{B}$ e os estímulos-escolha unitários A. Na Fase 4 (Teste AC-B), foram verificadas as relações emergentes entre os estímulos-modelo compostos $\mathrm{AC}$ (sendo $\mathrm{C}$ um áudio de 8 s de um trecho da música do compositor) e os estímulosescolha unitários B. Na Fase 5 (Teste C-A), foram verificadas as relações emergentes entre os estímulosmodelo unitários $\mathrm{C}$ e os estímulos-escolha A. Por fim, na Fase 6 (Pós-teste A-D), foram verificadas as relações emergentes estabelecidas entre estímulos-modelo unitários A e estímulos-escolha unitários envolvendo expressões faciais.

Em todas as tentativas (de treino e testes), era apresentado um estímulo-modelo e três estímulos-escolha. A cada tentativa, o participante clicava no estímulo-modelo e selecionava um dos três estímulos-escolha que apareciam lado a lado, embaixo do modelo. As apresentações dos estímulos foram aleatorizadas de maneira que os mesmos estímulos-modelo podiam se repetir em, no máximo, duas tentativas consecutivas. Todas as tentativas eram seguidas por um IET (Intervalo Entre Tentativas) de $2 \mathrm{~s}$. Em todas as fases, cada sessão foi composta por seis tentativas para cada estímulo-modelo, totalizando 18 tentativas por sessão. O critério de aprendizagem foi de 16 acertos em 18 tentativas. Caso o participante não cumprisse o critério, repetia-se a sessão apenas uma vez.

No treino (Fase 1), respostas de escolha do estímulo-escolha idêntico ao estímulo-modelo tinham como consequência o desenho de um sorriso (smile) e a adição de 10 pontos a um contador no canto superior esquerdo da tela. Para respostas de escolha do estímulo-escolha diferente do estímulo-modelo, a tela ficava branca. Os testes (Fases 2 a 6), por sua vez, foram realizados em extinção, de forma que as respostas não produziam consequências programadas, ou seja, independentemente da escolha feita, os estímulos desapareciam e a tela ficava branca. 
Tabela 1

Estímulos A, B, C e D Utilizados no Experimento

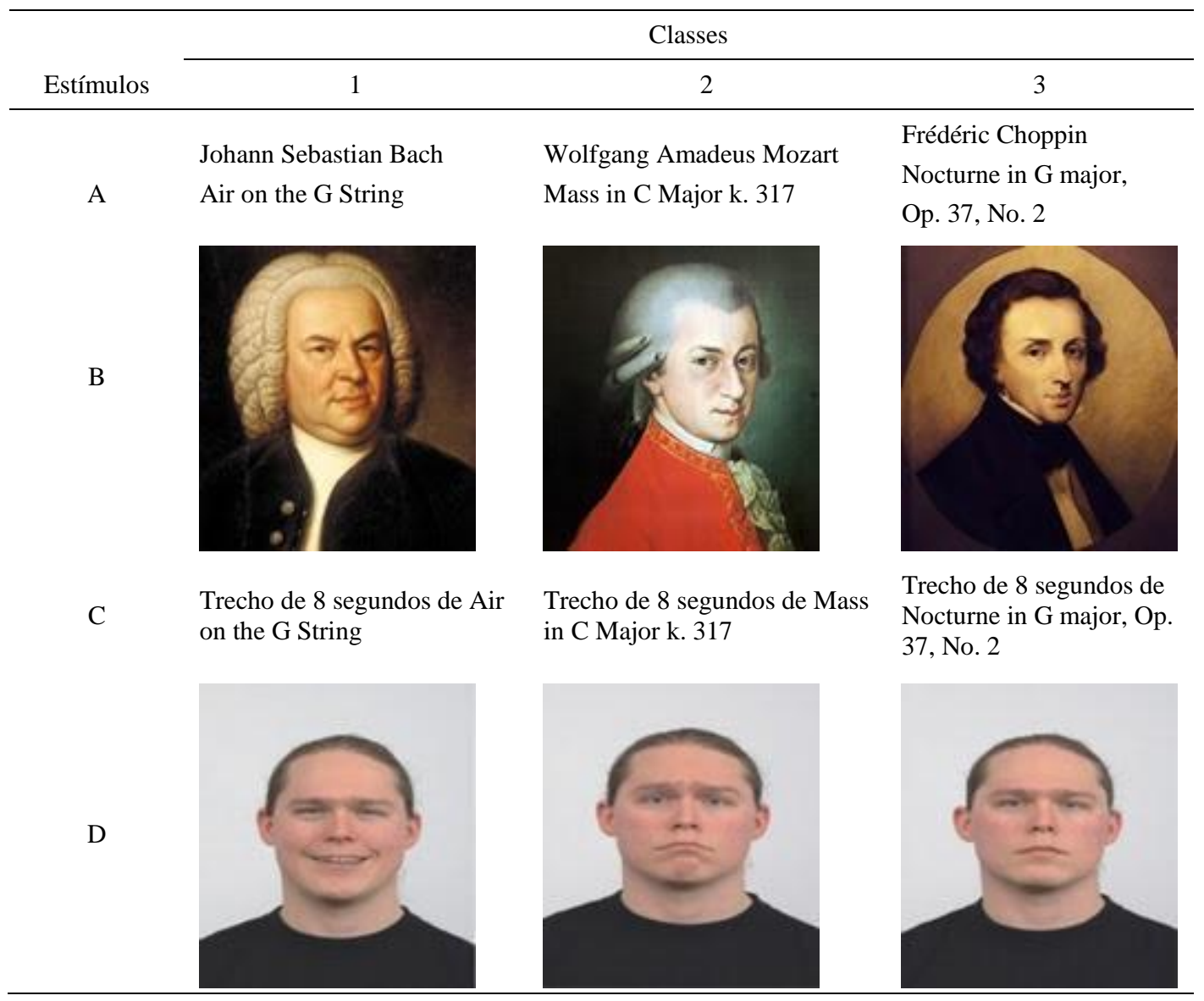

Durante a coleta, o participante sentava de frente para a tela do computador e poderia usar o mouse para movimentar o cursor e clicar em qualquer lugar da tela. No início de todas as fases, recebia instruções verbais do experimentador que aguardava o participante finalizar a fase do lado de fora da sala.

Fase 1: Treino $\boldsymbol{A B}-\boldsymbol{A B}: \mathrm{Na}$ Fase 1, foi realizado o treino de IMTS com estímulos compostos AB-AB. Os estímulos-modelo e os estímulos-escolha foram formados pelos elementos A e B, dispostos um ao lado do outro. Os três estímulos-modelo eram A1B1, A2B2 ou A3B3 e os estímulos-escolha poderiam ser A1B1, A1B2, A1B3, A2B1, A2B2, A2B3, A3B1, A3B2 e A3B3. As tentativas eram consideradas corretas caso o participante emitisse a resposta de clicar sobre o estímulo-escolha igual ao modelo, ou seja, diante de A1B1 selecionar A1B1, diante de A2B2 selecionar $\mathrm{A} 2 \mathrm{~B} 2$ e diante de A3B3 selecionar A3B3.

No início do treino, o participante recebia a instrução verbal do experimentador: "Você deve clicar no botão na parte superior da tela. Três opções aparecerão. Você deve escolher o estímulo igual ao estímulo apresentado na parte superior".

Fase 2: Teste A-B: Na Fase 2, foi realizado o teste das relações arbitrárias A-B para verificar se o treino de IMTS produziu relações condicionais emergentes entre os componentes A e B dos estímulos compostos. Os estímulos-modelo (A - nome do compositor e da música) e os estímulos-escolha (B foto do compositor) foram apresentados separadamente. No início dessa fase de teste, o participante recebia a instrução verbal: "Você deve clicar no botão na parte superior da tela. Três opções aparecerão. Você deve escolher a opção correspondente ao estímulo apresentado na parte superior".

Fase 3: Teste B-A:_A Fase 3 foi idêntica à Fase 2 , com a diferença de que foi realizado o teste das relações entre os estímulos B-A, ou seja, o estímulomodelo era B enquanto o estímulo-escolha era A. O objetivo foi verificar a relação emergente entre B e A.

Fase 4: Teste AC-B: Na Fase 4, os estímulos auditivos (trechos das músicas - Cs) foram apresentados juntamente com os estímulos As. Os estímulos-modelo foram compostos (A1C1, A2C2 e A3C3) e os estímulos-escolha foram unitários (B1, B2 e B3). Portanto, era possível responder corretamente apenas sob controle dos estímulos visuais A e B.

O participante deveria clicar sobre o botão com o estímulo-modelo visual (A). Essa resposta produzia o respectivo estímulo auditivo (C) por $8 \mathrm{~s}$, e os três estímulos-escolha (B), que apareciam lado a lado na parte inferior da tela. O participante deveria, então, selecionar B1, B2 ou B3 clicando no estímulo. O estímulo auditivo poderia ser reproduzido quantas vezes fossem necessárias até o participante selecionar um dos estímulo-escolha, o que finalizava a tentativa. 
O participante recebia a seguinte instrução do pesquisador: "Você deverá clicar sobre o estímulo que aparecerá na parte superior da tela. Você ouvirá uma música por $8 \mathrm{~s}$. Em seguida, escolha um dos três estímulos na parte inferior. Você pode ouvir a música quantas vezes quiser. Por favor, espere ela parar de tocar para selecionar o estímulo."

Fase 5: Teste $\boldsymbol{C}$-A: Na Fase 5, foi avaliada a emergência de relações condicionais C-A. Os estímulos-modelo eram unitários (C1, C2 ou C3 trechos das músicas), assim como os estímulos-escolha (A1, A2 e A3 - nomes dos compositores e das músicas). No início da tentativa, o participante clicava em um botão cinza, ouvia a música e clicava com o mouse em um dos estímulos-escolha que apareciam lado a lado na parte inferior da tela. Era possível reproduzir o estímulo auditivo quantas vezes fossem necessárias até a emissão da resposta que finalizava a tentativa.

O participante recebia a instrução verbal do experimentador: "Você deve clicar no botão em branco. Isso produzirá uma música, e as opções de escolha aparecerão. Você pode ouvir a música quantas vezes quiser, e depois deve escolher a opção correspondente à música que ouviu".

Fase 6: Pós-teste envolvendo expressões faciais: $\mathrm{Na}$ Fase 6, buscou-se observar se os participantes consistentemente escolheriam uma de três expressões faciais (estímulo-escolha D) diante de cada estímulo-modelo A formado por nomes dos compositores/títulos das obras. A cada tentativa, foi apresentado um estímulo-modelo unitário (A1, A2 ou A3) e, ao clicar sobre ele, eram apresentados três estímulos-escolha (D1, D2 e D3) lado a lado na parte inferior da tela. Após uma resposta de escolha de um dos estímulos Ds, iniciava-se a próxima tentativa.

O participante recebia a instrução verbal do experimentador: "Você deve clicar no botão na parte superior da tela. Aparecerão três opções. Você deverá escolher a opção correspondente ao estímulo apresentado na parte superior".

\section{RESULTADOS}

A Tabela 2 apresenta a porcentagem total de acerto dos participantes em cada fase do experimento. Todos os participantes concluíram o experimento em, no máximo, meia hora.

Todos os participantes atingiram o critério de acerto na primeira sessão de treino. $\mathrm{P} 4$ precisou de uma repetição do Treino $\mathrm{AB}-\mathrm{AB} \operatorname{logo}$ após a primeira sessão do Teste A-B, pois na primeira exposição ao teste a porcentagem de acerto foi de $27,78 \%$.

Os outros participantes (P1, P2 e P3) apresentaram $100 \%$ de acerto em uma única sessão do Teste A-B, indicando a emergência imediata dessas relações após o treino de IMTS.

No Teste B-A, todos os participantes apresentaram $100 \%$ de acerto na primeira sessão. Isso confirma a emergência dessas relações após o treino com o procedimento IMTS. No Teste AC-B, P2, P3 e P4 apresentaram 100\% de acerto. P1 apresentou 33,33\% de acerto. Esse participante escolheu sistematicamente B2 diante do modelo A1C1 e B1 diante de $\mathrm{A} 2 \mathrm{C} 2$, mantendo apenas as escolhas de $\mathrm{B} 3$ na presença de A3C3.

Tabela 2

Porcentagem Total de Acertos dos Participantes nas Fases do Experimento

\begin{tabular}{ccccc}
\hline Fases & P1 & P2 & P3 & P4 \\
\hline Treino AB-AB & $94,44 \%$ & $100 \%$ & $100 \%$ & $100 \%$ \\
Teste A-B & $100 \%$ & $100 \%$ & $100 \%$ & $100 \%^{\mathrm{a}}$ \\
Teste B-A & $100 \%$ & $100 \%$ & $100 \%$ & $100 \%$ \\
Teste AC-B & $33,33 \%$ & $100 \%$ & $100 \%$ & $100 \%$ \\
Teste C-A & $33,33 \%$ & $100 \%$ & $100 \%$ & $94,44 \%$ \\
\hline
\end{tabular}

Nota. ${ }^{a}$ P4 repetiu o treino uma vez após ter apresentado $27,78 \%$ de acertos no teste A-B. Após o retreino, obteve desempenho de $100 \%$, conforme descrito.

No Teste C-A, P2 e P3 obtiveram o número máximo de acerto (100\%). P4 teve apenas um erro $(94,4 \%$ de acerto). Para todos esses participantes, houve emergência de novas relações condicionais incluindo os estímulos auditivos C. P1 obteve apenas 33,33\% de acertos e respondeu de forma consistente com a fase anterior (C1-A2, C2-A1 e C3-A3).

$\mathrm{Na}$ última fase, no Pós-teste envolvendo expressões faciais, verificou-se que três dos quatro participantes relacionavam sistematicamente um dos estímulos Ds (expressão facial) a cada um dos estímulos As (previamente relacionados nos testes com os estímulos Cs - trechos de música).

De acordo com a Tabela 3, P1 escolheu o mesmo estímulo (D3 - expressão neutra) na maior parte das tentativas (9 em 18), independentemente do estímulo A apresentado. Esse desempenho, que envolveria um controle discriminativo simples, seria consistente com o desempenho apresentado nos testes anteriores levando-se em conta que para esse participante não foi verificada a formação de classes de equivalência (resultado negativo nos testes AC-B e C-A). P2, na maior parte das vezes, relacionou A1-D1, A2-D2 e A3-D3, exceto em duas tentativas nas quais relacionou A1-D2 e A2-D3. P3 e P4, sistematicamente, relacionaram A1-D3, A2-D2 e A3-D1 (somente em uma tentativa, P4 relacionou A3 com D3). Portanto, três dos quatro participantes sistematicamente escolheram determinadas expressões faciais a depender do estímulo modelo apresentado. Esses três participantes relacionaram D2 (expressão facial de tristeza) com A2 (nome - Mozart). P3 e P4 relacionaram A1 (nome - Bach) com D3 (expressão facial neutra) e A3 (nome - Choppin) com D1 (expressão facial de felicidade). Apenas P1 relacionou A1 (nome - Bach) com D1 (expressão facial de felicidade) e A3 (nome - Choppin) com D3 (expressão facial neutra). Portando, duas das relações condicionais estabelecidas foram iguais apenas para P3 e P4. 
Tabela 3

Quantidade de Respostas dos Participantes no Pós-teste (Fase 6) Envolvendo Expressões Faciais

\begin{tabular}{cccccccccccccc}
\hline Fase & & P1 & \multicolumn{1}{c}{ P2 } & \multicolumn{1}{c}{ P3 } & \multicolumn{3}{c}{ P4 } \\
\hline Pós-teste & D1 & D2 & D3 & D1 & D2 & D3 & D1 & D2 & D3 & D1 & D2 & D3 \\
\hline A1 & 1 & 2 & 3 & 5 & 1 & 0 & 0 & 0 & 6 & 0 & 0 & 6 \\
A2 & 1 & 2 & 3 & 0 & 5 & 1 & 0 & 6 & 0 & 0 & 6 & 0 \\
A3 & 3 & 0 & 3 & 0 & 0 & 6 & 6 & 0 & 0 & 5 & 0 & 1
\end{tabular}

Nota. Os dados sombreados em cor cinza se referem às respostas apresentadas em maior frequência pelos participantes.

\section{DISCUSSÃO}

A presente pesquisa buscou avaliar a possibilidade do procedimento de IMTS com estímulos compostos produzir relações condicionais emergentes envolvendo compositor/título, imagem do compositor e trecho das músicas como estímulos. Também pretendeu-se verificar se os participantes sistematicamente relacionariam alguma expressão facial ao nome do compositor/título da obra.

Os resultados indicaram a efetividade do procedimento utilizado para estabelecer relações condicionais emergentes para três dos quatro participantes. O que sugere que é possível estabelecer relações condicionais emergentes envolvendo trechos de música como estímulos a partir do procedimento IMTS com estímulos compostos, assim como ocorreu nos experimentos de Schenk (1993, 1995), Rebello et al. (2010), e Salvatori et al. (2012) que empregaram o mesmo procedimento, mas utilizaram estímulos visuais ou sonoros mais simples.

De forma mais abrangente, os resultados do presente experimento reiteram os resultados de outros estudos que revelam a utilidade do procedimento MTS para ensinar repertório simbólico, em especial, aqueles envolvidos no ensino de música (e.g., Hanna, Batitucci, \& Natalino-Ragel, 2016; Hanna, Huber, \& Natalino, 2016).

Mais especificamente, o presente estudo indica que o procedimento IMTS com estímulos compostos pode ser uma alternativa viável a ser empregada com sucesso para estabelecer aprendizagens simbólicas de forma rápida, com poucos ou nenhum erro, envolvendo estímulos sonoros mais complexos como aqueles presentes no ensino de ritmo, melodia, harmonia, entre outros. Crianças com desenvolvimento típico ou atípico poderiam se beneficiar desse procedimento no desenvolvimento de repertórios simbólicos musicais na medida em que o treino avaliado parte do ensino de relações de identidade que são muito mais facilmente estabelecidas do que o treino de relações condicionais arbitrárias para essa população. Nessa direção, futuros estudos poderiam verificar a efetividade do ensino de repertórios musicais complexos a partir de um treino com o procedimento IMTS para essa população.

No que diz respeito à avaliação da relação entre expressões faciais e os estímulos com nomes dos compositores, três dos quatro participantes relacionaram sistematicamente cada uma das três expressões faciais a um dos nomes dos compositores. O participante que não fez relações sistemáticas $(\mathrm{P} 1)$ foi o único que não demonstrou desempenhos indicativos de formação de classe de equivalência. Os outros três participantes sistematicamente relacionaram o estímulo D2 (expressão facial de tristeza) ao estímulo A2 (nome Mozart e título de uma de suas obras). Relações sistemáticas também foram estabelecidas pelos três participantes entre as demais expressões faciais e os nomes de compositores. P2 relacionou D1 com A3 e D3 com A1. P3 e P4 inverteram essas relações (D1-A1 e D3-A3). Portanto, esses resultados fornecem indícios que, talvez, o "significado" dos trechos das músicas para cada participante tenha se transferido para os estímulos As de cada classe de equivalência de modo que cada participante, que estabeleceu as classes de equivalência, pôde sistematicamente relacionar uma expressão facial a um dos estímulos envolvendo o nome do compositor sem que essa função tenha sido diretamente treinada.

Esses resultados iniciais podem servir de base para o desenvolvimento de futuros estudos que pretendam avaliar funções emotivas e sua transferência para estímulos arbitrários em classes de equivalência envolvendo estímulos sonoros. Emoções, como estímulos encobertos, são muitas vezes difíceis de serem identificadas por meio de sua expressão verbal e por serem produto de uma complexa história filogenética, ontogenética e cultural (Britto \& Elias, 2009; Skinner, 1945). O presente estudo parte da sugestão de Bortoloti e de Rose (2007) de que os "significados" das expressões faciais podem se transferir para outros estímulos da mesma classe de equivalência e fornece dados iniciais sobre novas formas de conduzir avaliação da transferência de funções emotivas que poderão ser aperfeiçoadas em estudos futuros. Sugere-se que futuros estudos exponham os participantes a uma avaliação inicial, antes do treino, de todos os estímulos empregados para averiguar quais expressões faciais são escolhidas diante de cada um desses estímulos. Dessa maneira, seria possível verificar se as escolhas das expressões faciais neutras seriam feitas para todos os estímulos antes do treino e se essas escolhas se modificariam ou não após formação das classes de equivalência. Dessa maneira, ter-se-ia maior controle experimental para se avaliar a transferência ou não das funções emotivas de músicas para estímulos que fazem parte da mesma classe de equivalência. 


\section{DECLARAÇÃO DE CONFLITO DE INTERESSES}

Os autores declaram que não há qualquer conflito de interesses relativos à publicação deste artigo.

\section{CONTRIBUIÇÃO DE CADA AUTOR}

Certificamos que todos os autores participaram suficientemente do trabalho para tornar pública sua responsabilidade pelo conteúdo. A contribuição de cada autor pode ser atribuída como se segue: Eloísa de Souza Fernandes, Natasha Takuno Hespanhol, Juliana Yu Ribeiro Toyoda e Viviane Nogueira da Silva foram responsáveis pela elaboração do projeto e coleta de dados, e contribuíram com a revisão bibliográfica, tratamento de dados, discussão dos resultados e conclusão. Paula Debert e Alceu Martins Filho foram responsáveis pela definição da metodologia e contribuíram com a revisão bibliográfica, programação do software utilizado, tratamento dos dados, discussão dos resultados e conclusão. Eloísa de Souza Fernandes, Natasha Takuno Hespanol, Alceu Martins Filho e Paula Debert foram responsáveis pelo processo de revisão e redação final.

\section{DIREITOS AUTORAIS}

Este é um artigo aberto e pode ser reproduzido livremente, distribuído, transmitido ou modificado, por qualquer pessoa desde que usado sem fins comerciais. $\mathrm{O}$ trabalho é disponibilizado sob a licença Creative Commons 4.0 BY-NC.

\section{(cc) BY-NC}

\section{REFERÊNCIAS}

Bachorik, J. P., Bangert, M., Loui, P., Larke, K., Berger, J., Rowe, R., \& Schlaug, G. (2009). Emotion in motion: Investigating the time-course of emotional judgments of musical stimuli. Music Perception: An Interdisciplinary Journal, 26(4), 355-364. http://doi.org/10.1525/mp.2009.26.4.355

Bortoloti, R., \& de Rose, J. C. (2007). Medida do grau de relacionamento entre estímulos equivalentes. Psicologia: Reflexão e Crítica, 20(2), 250-256. http://dx.doi.org/10.1590/S0102-79722007000200011

Britto, I. A., \& Elias, P. V. O. (2009). Análise comportamental das emoções. Psicologia para América Latina, 16. Disponível em: http://pepsic.bvsalud.org/scielo.php?script=sci_arttext \&pid=S1870-350X2009000100004\&lng=pt\&tlng=pt.

Cumming, W., \& Berryman, R. (1965). The complex discriminated operant: Studies of matching-to-sample and related problems. In: D. I. Mostofski (Ed.), Stimulus generalization, (pp. 284-330). Stanford: Stanford University Press.

Hanna, E. S., Huber, E. R., \& Natalino, P. C. (2016). Aprendizagem de rudimentos de leitura musical com ensino cumulativo e não cumulativo de relações condicionais. Psicologia: Teoria e Pesquisa, 32(5). https://dx.doi.org/10.1590/0102-3772e32ne25.

Hanna, E. S, Batitucci, J. S. L., \& Natalino-Rangel, P. C. (2016). Paradigma de equivalência de estímulos norteando o ensino de rudimentos de leitura musical.
Acta Comportamentalia, 24 (1), 29-46. Retirado de: http://www.revistas.unam.mx/index.php/acom/article/v iew/54711/48627.

Langner, O., Dotsch, R., Bijlstra, G., Wigboldus, D. H. J., Hawk, S. T., \& van Knippenberg, A. (2010). Presentation and validation of the Radboud Faces Database. Cognition \& Emotion, 24(8), 1377-1388. http://doi.org/10.1080/02699930903485076

Markham, M. R., \& Dougher, M. J. (1993). Compound stimuli in emergent stimulus relations: Extending the scope of stimulus equivalence. Journal of the Experimental Analysis of Behavior, 60(3), 529-542.

Mohn, C., Argstatter, H., \& Wilker, F. W. (2011). Perception of six basic emotions in music. Psychology $\begin{array}{lll}\text { of } \quad \text { Music, } & \text { 39(4), }\end{array}$ https://doi.org/10.1177/0305735610378183

Rebello, M., Yu, L. S., Bertoldo, M., Duckur, R., Vernucio, R., \& Debert, P. (2010). Ensino de ideogramas com base no procedimento matching de identidade com estímulos compostos. Revista Brasileira de Análise do Comportamento, 6(2), 203210. http://dx.doi.org/10.18542/rebac.v6i2.1119

Salvatori, A., Silva, C. dos S., Belem, I. E. de A., Modenesi, R. D., \& Debert, P. (2012). Matching de identidade com estímulos compostos e o ensino de notas musicais. Acta Comportamentalia, 20(3), 287298.

Schenk, J. J. (1993). Emergent conditional discrimination in children: Matching to compound stimuli. The Quarterly Journal of Experimental Psychology, 46(4), 345-365. http://doi.org/10.1080/14640749308401076

Schenk, J. J. (1995). Complex stimuli in nonreinforced simple discrimination tasks: Emergent simple and conditional discriminations. The Psychological Record, 45(3), 477-494. http://doi.org/10.1007/BF03395156

Sidman, M. (1994). Equivalence relations and behavior: A research story. Boston: Authors Cooperative.

Sidman, M. (2000). Equivalence relations and the reinforcement contingency. Journal of the Experimental Analysis of Behavior, 74(1), 127-146. http://doi.org/10.1901/jeab.2000.74-127

Skinner, B. F. (1945). The operational analysis of psychological terms. Psychological Review, 52(5), 270-277. http://doi.org/10.1037/h0062535

Stromer, R., \& Stromer, J. B. (1990). The formation of arbitrary stimulus classes in matching to complex samples. The Psychological Record, 40(1), 51-66. http://doi.org/10.1007/BF03399571

Submetido em: 13/07/2018 Aceito em: 02/02/2019 